\title{
Mobile Virology Research and Diagnostic Laboratory (MVRDL: BSL-3) for COVID-19 Screening, Virus Culturing and Vaccine Development
}

\author{
Yellamanchali Sreenivas Rao ${ }^{1}$
}

Received: 8 May 2020 / Revised: 11 June 2020 / Accepted: 29 June 2020 / Published online: 4 July 2020

(C) Indian National Academy of Engineering 2020

\begin{abstract}
In order to prevent spread of COVID-19, World Health Organization (WHO) has specified that measures such as cleaning hands regularly with alcohol-based hand sanitizer or washing with soap and water, avoiding touching nose, eyes, mouth and social distancing should be followed by people. Another important measure for containing spread is by Testing-Testing and Testing. By testing, patients affected by COVID-19 can be isolated and further spread from them can be prevented. For testing, clinical specimen inactivation should be performed in a bio-safety level-3 (BSL-3) laboratory and after this step, routine testing can be performed in a bio-safety level-2 laboratory. Based on the infection and death rates in the current pandemic situation, few high-level bio-safety facilities are required to handle this deadly virus associated with potential exposure risks. It would take a long time, a large staff and many resources to construct a new fixed bio-safety facility, thus delaying the prevention and control of the pandemic. Therefore, a mobile BSL-3 laboratory with both bio-safety and flexibility is urgently needed to manage the pandemic and emergent public health incidents. To meet this requirement, the author has designed a mobile virology research and diagnostic laboratory with inputs from ESIC Medical College, Hyderabad, identified two companies which specialize in fabrication of shelters and containment facilities, realized and installed it within 15 days of time. This state-of-the-art, one of its kind laboratory was inaugurated by Hon'ble Raksha Mantri on 23 April, 2020. The MVRDL developed as per WHO and ICMR bio-safety standard (BSL-3) can be used:
\end{abstract}

- To conduct real-time reverse transcription polymerase chain reaction (rRT-PCR) test for diagnosing COVID-19. Possibility to test up to 2000 samples per day.
- For virus culturing for drug screening, convalescent plasma-derived therapy.

- To aid in the development of vaccine and development of diagnostic kits.

Keywords COVID-19 $\cdot$ Bio-safety $\cdot$ Mobile bio-safety laboratory $\cdot$ Containment

\section{Introduction}

World Health Organization (WHO) declared a Public Health Emergency of International Concern on 30 January, 2020 and named the disease caused by SARS-CoV-2 virus as COVID-19. This disease is spreading globally, and it is very important to take necessary measures to contain it. The rapidly spreading, highly contagious and pathogenic SARS-coronavirus 2 (SARS-CoV-2) is transmitted

Yellamanchali Sreenivas Rao

ysr@rcilab.in

1 Project Director, AD(FV-Exo), PGAD/DRDO, Kanchanbagh P.O, Hyderabad 500058, India human-to-human has continuously spread to 200 countries. It is must to immediately build, expand, train and deploy health workers to find, test, isolate and treat every case and trace every contact. The two most important measures to prevent infection are social distancing and Testing-Testing and Testing.

Clinical specimen inactivation should be performed in a bio-safety level-3 (BSL-3) laboratory (negative pressure, HEPA filters, anteroom and self-locking doors) and after this step, routine testing can be performed in a bio-safety level-2 laboratory. However, the pathogenicity of COVID-19 is not completely known yet, therefore BSL-3 lab is recommended for samples processing. Based on the infection and death rates, few high-level bio-safety facilities are required 
to handle this deadly virus associated with potential exposure risks. It would take a long time, a large staff and many resources to construct a new fixed bio-safety facility, thus delaying prevention and control of the pandemic.

Therefore, a mobile BSL-3 lab with both bio-safety and flexibility is urgently needed to manage pandemic and emergent public health incidents. The author has adopted the technology of containerization of ground support equipment for missiles and clean rooms technology for integration of high-precision missile components to suit the bio-safety standards and has come up with a solution for establishing COVID-19 diagnostic laboratory. To that end, a mobile virology research and diagnostic laboratory was designed with inputs from ESIC Medical College, Hyderabad. Two companies in Hyderabad namely, M/s ICOMM which specializes in fabrication of shelters and M/s iClean Technologies, a turnkey design and build Company with years of experience in building the containment facilities were identified for manufacturing of this lab.

In addition to screening, the lab can also be used for conducting research projects related to drug discovery, therapeutics and vaccine development in collaboration with National and International organizations. Very importantly, BSL3 lab is mandatory for live virus cultures and having this facility is the main criterion to carryout research projects. Outbreaks of viral agents are frequent in India. Timely diagnosis of these outbreaks is critical to mount appropriate public health response. The inadequacy leads to epidemics, for example, SARS and CORONA, where we need infrastructure (Biosafety level 2 or 3 ) for handling viruses, diagnostics facility to cater patients from all over India.

\section{Design and Implementation}

All the necessary equipments for meeting objectives mentioned above are configured in two mobile containers as shown in the following schematics (Fig. 1).

The clinical specimen inactivation is to be performed in a Bio-Safety Level-3 laboratory (negative pressure, HEPA filters, anteroom and self-locking doors) and subsequent to this step, routine testing is to be performed in a Bio-Safety Level-2 Laboratory.

The entire area is conditioned with HVAC system with a design of $100 \%$ fresh air ensuring no re-circulation. Room temperature of $24 \pm 4{ }^{\circ} \mathrm{C}$ is maintained, relative humidity is maintained less than $60 \%$ as per specifications of BSL-3 and BSL-2 labs.

The system is equipped with access control, LAN, telephone cabling and CCTV. For maintaining safety fire alarm system is provided. The system has built-in furniture and is equipped with pass boxes for transfer the samples from one container to other container. Personnel protective equipment is provided for personnel to cross over box.

\section{Process Flow for Virus Diagnostics}

\section{Personnel Movement in BSL-III}

The person enters the change room and wears the BSL-3 lab suits and enters the lab through ante room. After completion of activities move to mist shower via ante room. After mist shower cycle, will remove BSL-3 lab suits/shoes leaves them in the appropriate place provided in shower.

\section{Sample Movement}

The samples will be transported in viral culture medium to the lab. Then they will be placed in dynamic pass box (DPB 1) to restrict the man movement inside the lab. Technician in BSL3 lab (Lab 1) will collect the sample and process in bio-safety cabinet Class II type A2 and B2 using neutralization buffer. After neutralization of virus, cell pellet will be collected in Eppendorf tubes and transferred to BSL2 lab (Lab 2) through the dynamic pass box (DPB2) for screening the virus using RT-PCR machine.

\section{Bio-waste, Solid Waste and Liquid Waste}

The bio-waste and solid waste of BSL-III \& BSL-II labs will be packed in container suitable for autoclaving and will be sent out of the lab through Dunk tank to ensure surface decontamination. Accordingly, in the lab, the generated liquid waste are sent through the sink provided in the lab and will be collected in a liquid waste collection tank provided outside the container lab through piping by gravity. The tank is filled with chemical decontamination agent and the contaminated liquids gets decontaminated chemically and further shall be sent for appropriate treatment. Inside pictures of some portions of MVRDL are shown in Fig. 2.

\section{Resources}

DRDO has designed the laboratory with the inputs provided by ESIC Medical College, Hyderabad. The following industrial partners participated in realization of the MVRDL Lab:

- $\mathrm{M} / \mathrm{s}$ iCOMM, Hyderabad has provided two containers.

- $\mathrm{M} / \mathrm{s}$ iClean, Hyderabad has built the containment facilities with required safety levels.

- M/s Hitech Hydraulics, Hyderabad has provided foundation structure. 
Fig. 1 Layout of MVRDL
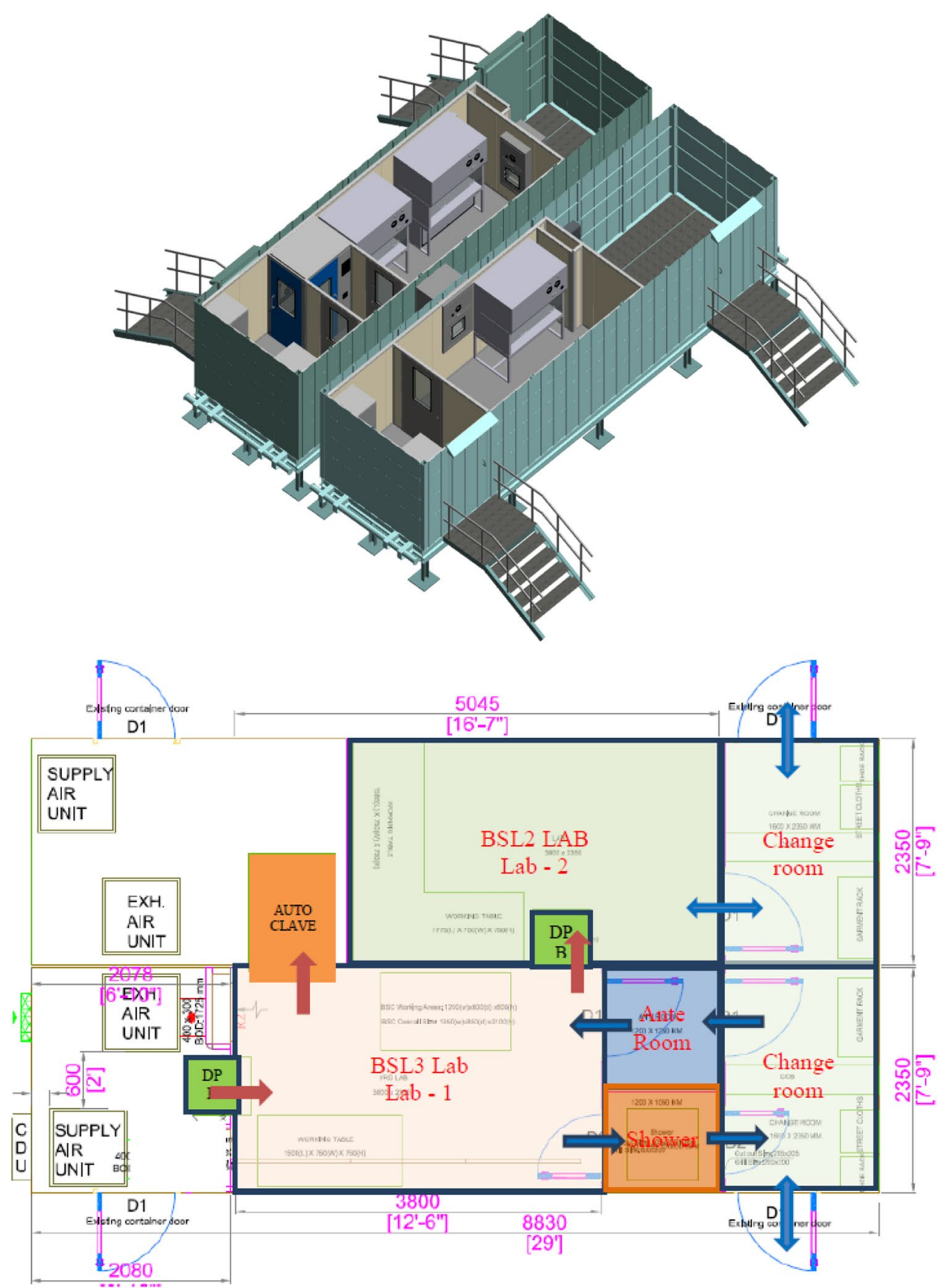

\section{Results and Discussion}

The author has adopted the technology of containerization of ground support equipment for missiles and clean rooms technology for integration of high-precision missile components to suit bio-safety standards and has come up with a solution for establishing COVID-19 diagnostic laboratory in a mobile container meeting the bio-safety standards (BSL-3 \& BSL-2). This laboratory is designed to carry out following activities: (a) To screen up to 2000 samples per day

(b) To carryout drug screening

(c) Therapeutics

- Convalescent plasma therapy

- Therapeutic value of milk exosomes for COVID-19

- Stem cell therapy to reduce inflammation and fibrosis

(d) COVID-19 diagnostics 


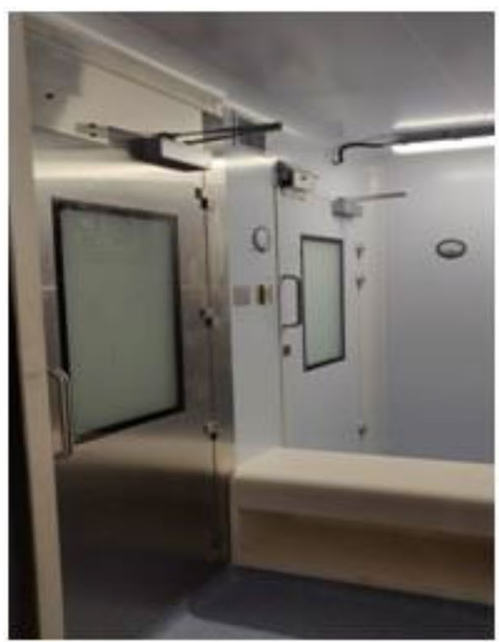

Change Room

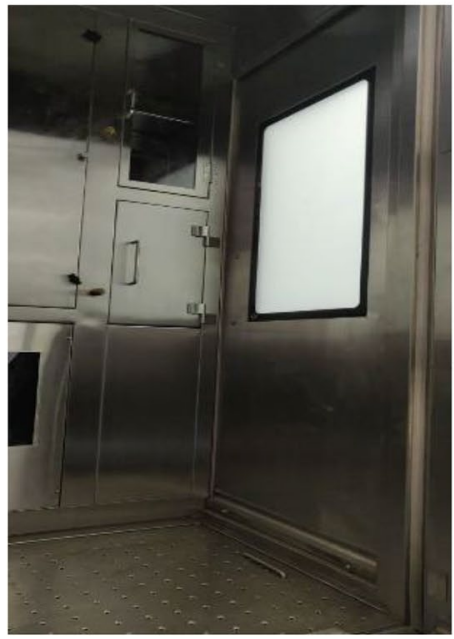

Shower

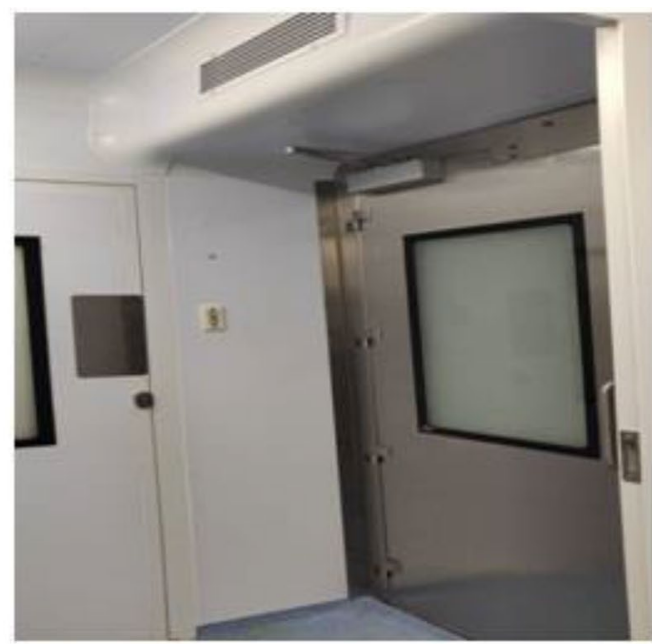

Ante Room

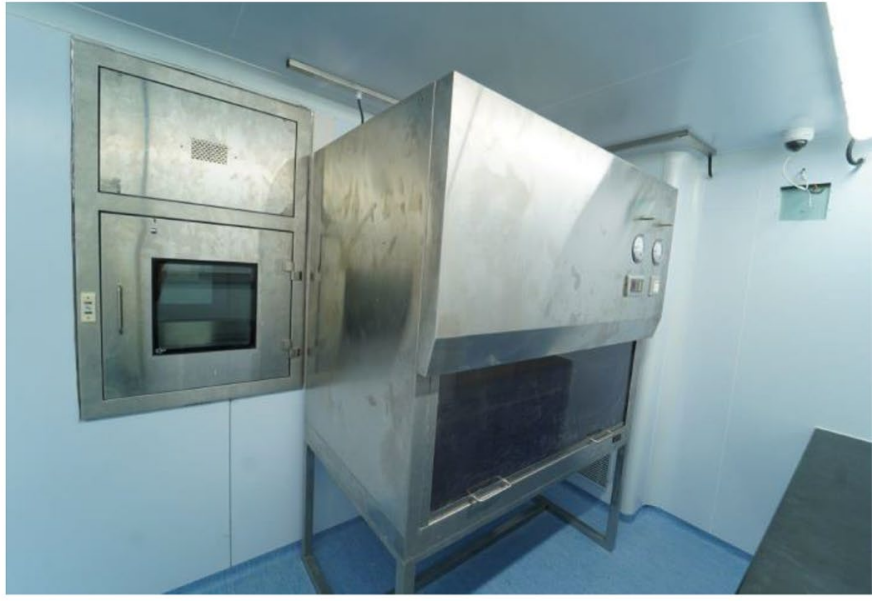

Dynamic Pass Box

Fig. 2 Inside pictures of some portions of MVRDL

- Covid $i$-Chip-A simple, fast and highly sensitive detection of asymptomatic SARS COV2

- $\operatorname{IgM} / \operatorname{IgG}$ - Rapid antibody test

- Single-tube PCR test using isothermal amplification

- Direct sequencing of corona viruses from clinical specimens, using nanopore digital target selection.

(e) Vaccine development

- Comprehensive immune profiling of COVID-19 patients towards vaccine design and early clinical trials specific to Indian population.

\section{Conclusion}

The mobile virology research and diagnostic laboratory, which has a capability to handle up to 2000 SARS-COV-2 virus samples in a day, maintaining BSL-3 standards is designed, realized and installed at ESIC Medical College, Hyderabad, within a time frame of 15 days. This is a first of its kind mobile diagnostic laboratory that can be transported and rapidly deployed at COVID-19 hotspots in any corner of our country. This laboratory can also aid in virus culturing for drug screening, convalescent plasma-derived therapy and development of diagnostic kits. 


\section{Reference}

https://www.who.int/news-room/detail/30-01-2020-statement-on-thesecond-meeting-of-the-international-health-regulations-(2005)emergency-committee-regarding-the-outbreak-of-novel-coron avirus-(2019-ncov). Accessed 1 July 2020
Publisher's Note Springer Nature remains neutral with regard to jurisdictional claims in published maps and institutional affiliations. 